\title{
The Analysis on the Effect of Passenger car Air Conditioning and Distribution with Different Inlet Parameters Hao Yang ${ }^{1}$, Yidong Wang ${ }^{2}$, Taibi He ${ }^{2}$ \\ ${ }^{1}$ Chengdu Textile College, Chengdu Sichuan 611731, China; \\ ${ }^{2}$ XiHua University, Chengdu Sichuan 611743, China
}

\begin{abstract}
Keywords: Fluent; Passenger car; Thermal environment; Air distribution
\end{abstract}
\begin{abstract}
With the development of vehicle industry and the improvement of life quality, many factories and scholars are more focused on heating facility and air quality of the Passenger car.This paper utilizes Fluent analysis software, adopting RNG k- $\varepsilon$ and DTRM radiation heat transfer to make a three-dimensional numerical model.This paper is for the Passenger car indoor air distribution,which is analyzing effect pattern of inlet parameters for temperature field and velocity field.This paper has providing a theoretical basis for improvements of indoor thermal environment and heating comfort.It is also the guidance for the optimal design about vehicle air conditioning and distribution.
\end{abstract}

\section{Introduction}

At present, the design of the automobile air conditioning system is mainly to car internal heating load for criteria, and ignoring the car interior department structure and different air supply parameters on the effect to air distribution and air quality. The thermal comfort of passengers depends on the internal environment and the way of air distribution, the unreasonable airflow organization is easy to cause airflow corner or gas resistance[1], the local temperature is too high or too low, which will cause energy waste and influence thermal comfort of passengers.

Different supply air velocity and temperature on the car chamber has a great influence on air distribution characteristics. Vehicle indoor air velocity and temperature on the space has the magnetic field characteristics. In different times and different regions have different values. The different areas of the car interior with different thermal comfort [2].Therefore, the characteristics of vehicle indoor air distribution and thermal comfort study of rational design best ventilation scheme, improve internal environment has important practical significance. It is also the foundation of evaluation car internal environment.

\section{Model establishment}

Based on some brand domestically produced 37 travel bus as the research object, the rear engine, outer contour size $8995 * 2380 * 2380(\mathrm{~mm})$, the bus is a form of on the air supply on return air decorate, both side duct is located in the roof, the supply air duct outlet along the duct length direction uniformly distributed in the upper part of the seat rows, return air mouth arranged in the middle of the car is. When modeling in precision and calculation results under the condition of little effect, can do to study appropriate simplified[3].The physical model grid.

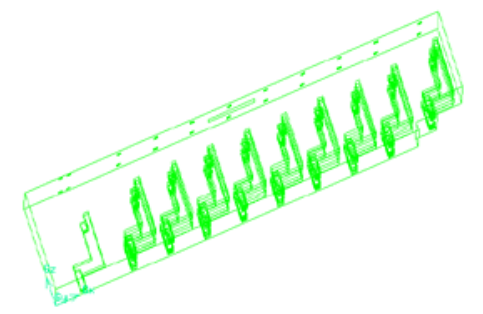

Fig.1:The geometrical model of passenger car 


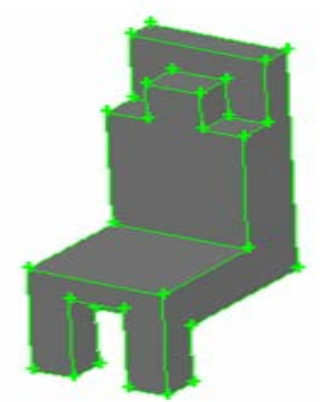

Fig.2: The seat body model diagram

\section{Boundary conditions set}

This paper adopted $R N G K-\varepsilon$ turbulence model and discrete DTRM in thermal radiation model, boundary conditions are set to the velocity - inlet, the hydraulic diameter of $0.08 \mathrm{~m}$, all the Air supply outlet will be supplied. Outlet is set to the pressure - outlet, the hydraulic diameter is 0.571 $\mathrm{m}$. The second class boundary conditions of car body wall heat and solar radiation unified simplified as bodywork uniform incoming wall heat flux through the body surface to car interior [4].Engine room temperature is taken $333.15 \mathrm{~K}$, car body surface heat transfer coefficient is taken $10 \mathrm{w} / \mathrm{m}^{2} \cdot k$. Human body Heat source with constant Heat Flux boundary condition, the Heat Flux of $20 \mathrm{w} / \mathrm{m}^{2}[5]$.

\section{Analysis of the results}

Car room with longitudinal symmetry the characteristics, to reduce the amount of calculation, this article only in longitudinal direction take half of the vehicle as the research object. In order to analyze the influence of different air distribution parameters on the passenger flow distribution, this article selects several special section as monitoring surface, for monitoring the surface temperature field and velocity field were analyzed. $X=6.83 \mathrm{~m}$ vertical section is said by 7 seat rows of air supply outlet above the plane, $\mathrm{Y}=0.8 \mathrm{~m}$ section is said by the middle aisle side longitudinal plane passengers area, $x=4.43 \mathrm{~m}$ row seat the intersection of plane perpendicular to the air supply outlet and inlet, $\mathrm{z}=1.05$ section plane passengers facial area.

\subsection{The impact of different supply air speed for the car indoor air distribution}

In order to analyze the influence of different supply air speed for the car indoor air distribution, this paper adopts the supply air temperature is $293.15 \mathrm{~K}$, the supply air velocity V respectively 2 , 3, 4, $5 \mathrm{~m} / \mathrm{s}$ four kinds of air conditioning air supply.

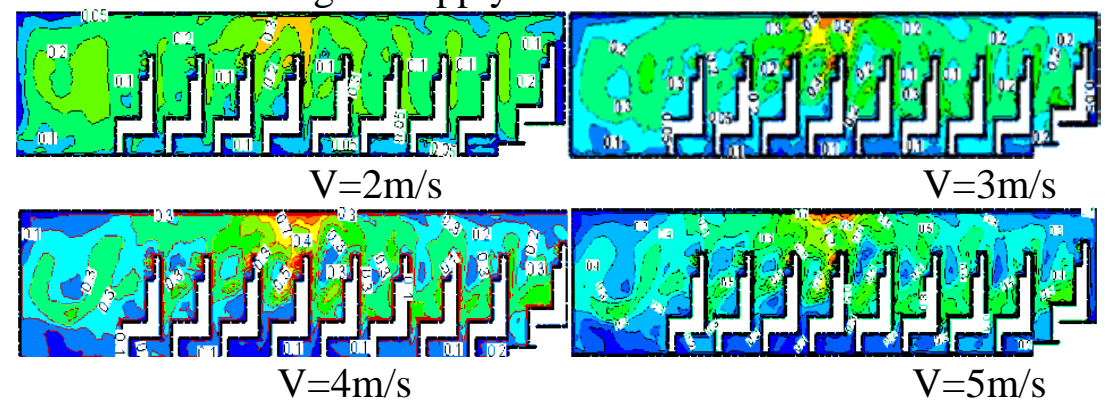

Fig.3: Different supply air speed distribution at $\mathrm{Y}=0.8$

The experimental results show that the summer in-car air velocity of $0.3 \sim 0.4 \mathrm{~m} / \mathrm{s}$, the body is more comfortable[6].In fig.3, the velocity field under different supply air speed distribution is different, with a gradual increase in supply air velocity, bus and coach interior each longitudinal section of the wind speed increased obviously,. When the supply air velocity is $2 \mathrm{~m} / \mathrm{s}$ and $3 \mathrm{~m} / \mathrm{s}$, passengers face air velocity will be around $0.1 \sim 0.2 \mathrm{~m} / \mathrm{s}$, facial airflow speed is too low. Under this 
situation no passengers have blowing feeling, and they will feel very hot; When the supply air velocity of $4 \mathrm{~m} / \mathrm{s}$, and passengers face air velocity will be around $0.3 \mathrm{~m} / \mathrm{s}$. Under this situation the air velocity is good - facial hair drier are moderate , and passengers feel cool. If the supply air velocity is $5 \mathrm{~m} / \mathrm{s}$, the air velocity will be too big and the wind is too strong.
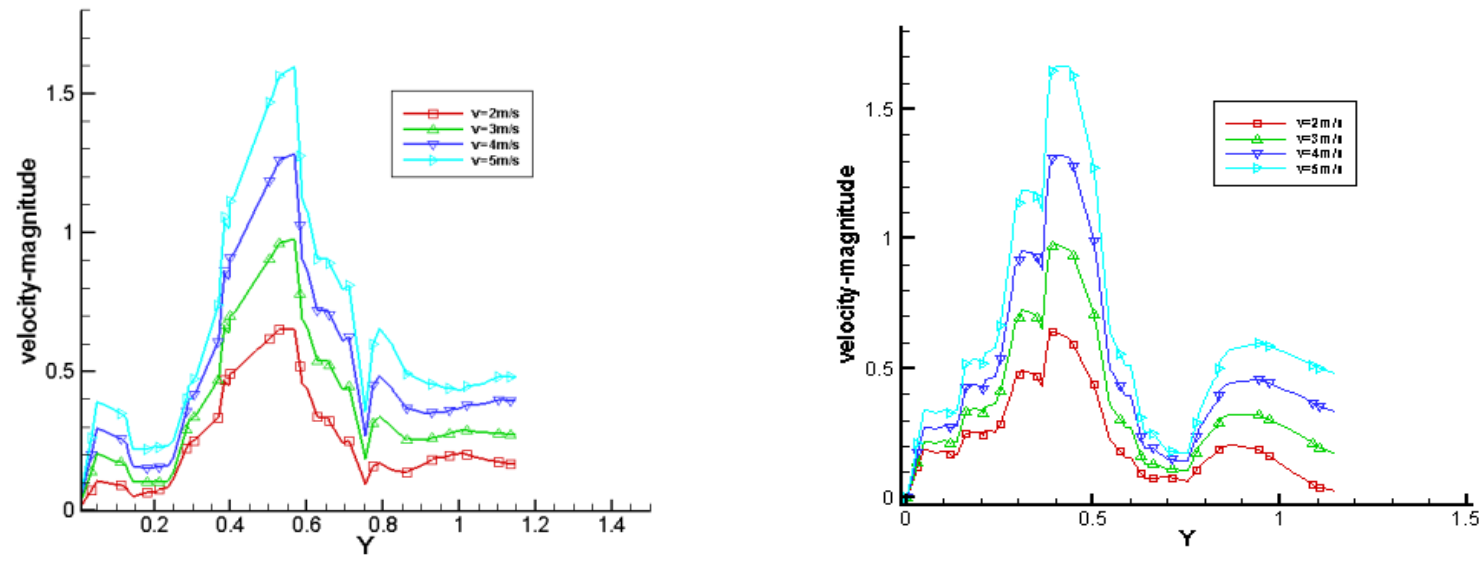

Fig.4: Speed curve at intersecting line of $X=4.43 、 X=6.83$ with $Z=1$.

In fig.4, the speed curve is almost the same. It show that different supply air speed of vehicle indoor have little influence. However, the airflow velocity near $\mathrm{Y}=1 \mathrm{~m}$ inflection point, In figure left ,the change speed trend is relatively flat, changing trend. In figure right ,the rate fell sharply, which indicates that the position of return air mouth has certain influence on the velocity distribution characteristics. If near the inlet area of passengers flow faster is helpful for ventilation. But air velocity away from the inlet area is weak.
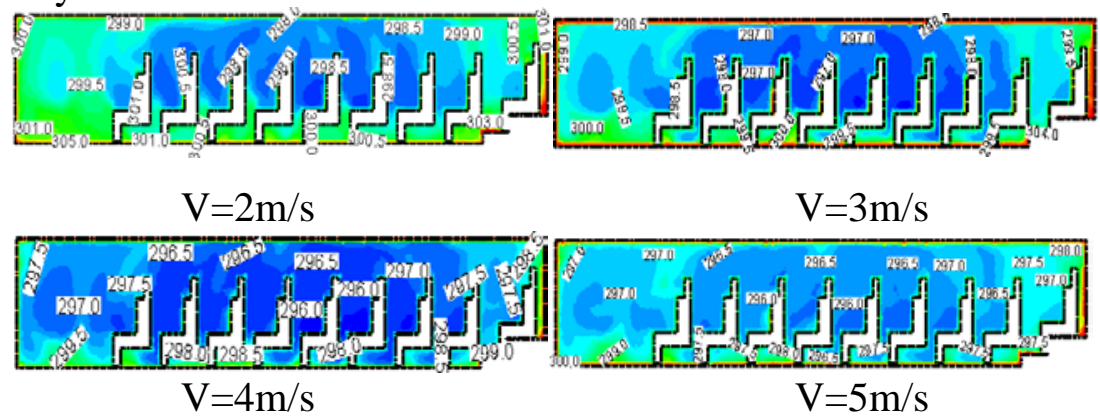

Fig.5:Temperature distribution at different wind speed with cross section $\mathrm{x}=6.8$

In fig.5, with the increase of wind speed, cold quantity will increase in per unit time and the car indoor temperature will decrease .Car room before and after the end by the large area of the wind window solar radiation and engine room temperature, the influence of temperature is higher than middle area $1 \sim 2 \mathrm{k}$, thermal comfort is poorer, can consider to increase cooling capacity in the region. When $\mathrm{V}=2 \mathrm{~m} / \mathrm{s}$, the cross section of most of the temperature between $298.5 \sim 301 \mathrm{k}$. If the temperature gradient in the direction of car room height is larger ,and the temperature uniformity is poor; When $\mathrm{V}=3 \mathrm{~m} / \mathrm{s}$, the cross section of most of the temperature in the range of $297 \sim 298.5 \mathrm{~K}$. If temperature uniformity, the temperature will be range to satisfy the thermal comfort of most of the passengers and the seat beneath the cold air is not easy to achieve. It will lead to weak turbulence intensity and higher temperature; When $\mathrm{V}=4 \mathrm{~m} / \mathrm{s}$ and $\mathrm{V}=5 \mathrm{~m} / \mathrm{s}$, the car indoor temperature will be low and poor thermal comfort of passengers.

\subsection{The impact of supply air temperature for the indoor air distribution}

In the same way, in order to analyze the impact of supply air temperature in passenger indoor air distribution, this paper adopt the rate of $4 \mathrm{~m} / \mathrm{s}$ air supply and air supply temperature $\mathrm{T}$ were 291.15, 293.15, 295.15, 297.15 K as four different air conditioning air supply condition. 


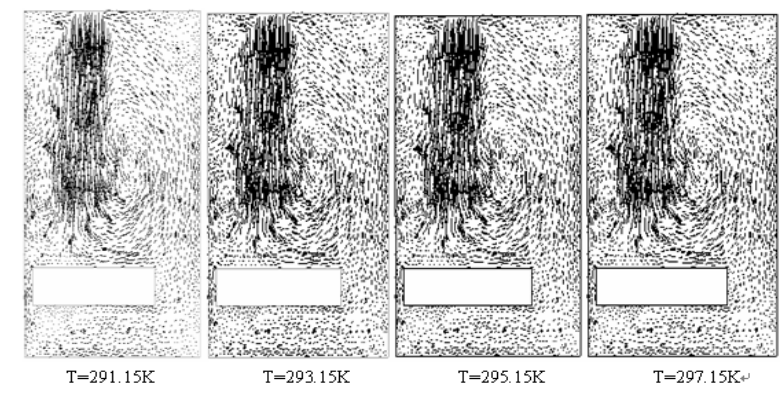

Fig.6: velocity vector at different supply air temperature with cross section $\mathrm{x}=6.83$
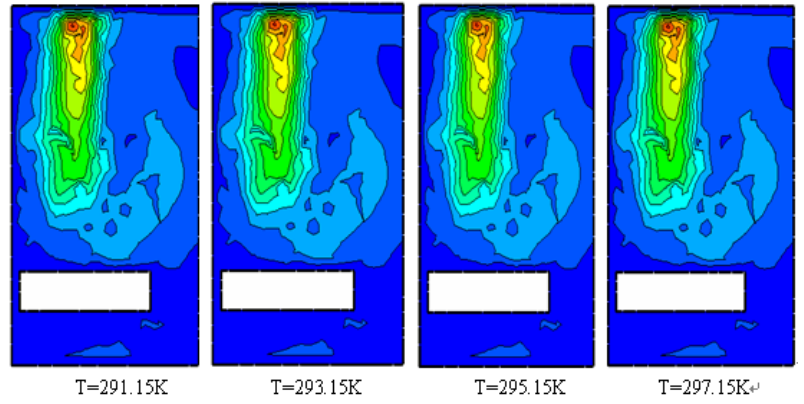

Fig.7: distribution of velocity field at different supply air temperature with cross section $x=6.83$

Fig.6 and fig.7, when the supply air velocity is constant, we only change the supply air temperature. The cross section of $\mathrm{X}=6.83$ on airflow pattern basically remain unchanged, that is, within the scope of small temperature difference, we only change the supply air temperature. The car indoor air flow pattern basic has no effect .The speed of passenger car interior each location field distribution is almost the same.
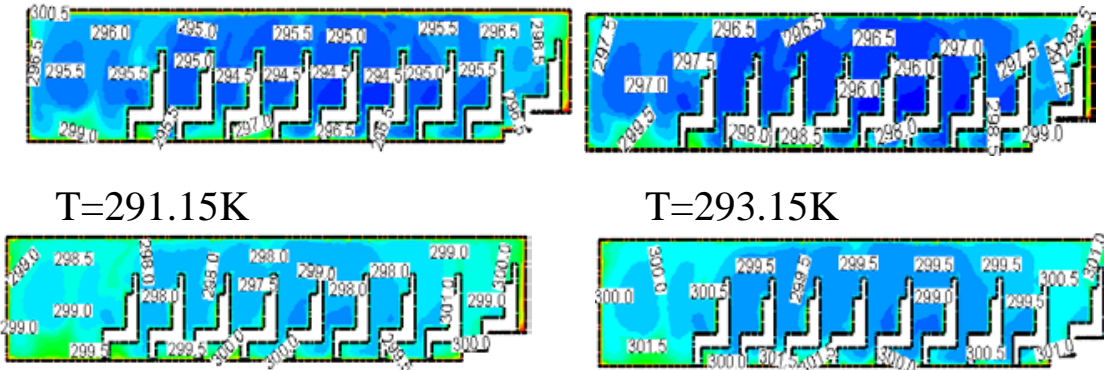

$\mathrm{T}=295.15 \mathrm{~K}$

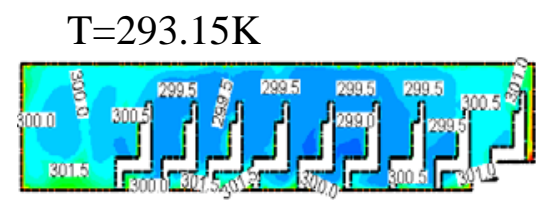

$\mathrm{T}=297.15 \mathrm{~K}$

Fig.8: Temperature distribution at different supply air temperature with cross section $\mathrm{Y}=0.8$

Fig.8, with the increase of supply air temperature, the amount of cold that into the car at unit time will reduce, and the car indoor temperature rise gradually. Due to the influence of larger solar radiation and

The engine temperature, the temperature of Car front-end and back-end is higher. These two areas had a great influence on the uniformity of temperature distribution inside the car. We can take different amounts air supply to improve temperature uniformity. When the supply air temperature is 291.15K, and most temperature section is located in the range of $294.5 \sim 295.5 \mathrm{~K}$.It will make passengers feel cool and comfortable ; When the supply air temperature is $295.15 \mathrm{~K}$, most cross section temperature between $297.5 \sim 298.5 \mathrm{~K}$, this temperature basically achieve thermal comfort of the human body; When the supply air temperature is $297.15 \mathrm{~K}$, facial area temperature $299.5 \mathrm{~K}$, the interior temperature is higher so that passengers will feel even more hot.

\section{Conclusion}

In this paper, based on the research of the coach indoor air flow characteristics, we can draw the following conclusions: (1) the peripheral structure, Windows, the engine room have larger heat load on the car interior, and its near temperature distribution is significantly higher than other area. 
Therefore, it is necessary to improve the heat insulation effect of peripheral structure (including windows) or increase the amount of cold in these special areas.(2)The influence of different supply air speed of air form small supply air speed 、 air flow intensity increases and the vortex intensity increase. When the air conditioning supply air velocity $\mathrm{V}=4 \mathrm{~m} / \mathrm{s}$, passengers wind feels more appropriate facial area and the body is more comfortable.(3) Different supply air temperature on the temperature field had a greater influence and velocity field have little impact. When the supply air temperature is $295.15 \mathrm{~K}$, much of the car indoor temperature can satisfy the requirement of the thermal comfort of human body.(4) The distribution of return air supply outlet has certain influence to temperature field. The velocity field air diffuser and return air velocity distribution near the mouth is higher than other area. However, the temperature is lower than other area.

\section{References}

[1]Zhang Kangzhi, Wangfang etc, Double support of vortex pump performance analysis and the analysis of Fluent flow[J].Manufacturing automation, 2014.03:47-49.

[2]Kenji Furuse, Thoru Kornoriya.Study of passenger's comfort in non-uniform thermal environments of vehicle compartment [J]. JSAE Review, 1997, 18(4): 411-414.

[3]Chow W K. Ventilation of enclosed train compartments in Hong Kong[J]. Applied Energy, 2002, 71(3): 161-170.

[4]Che Yanqiu, Analysis of car indoor air flow field and temperature field of numerical[D].Liaoning Province: Dalian University of Technology

[5] Yang Juan, Numerical simulation of car air conditioning indoor air flow organization[D].Shan Dong: Qing Dao University,2006.

[6]Jin Zhangyong, Experimental study and numerical simulation of airflow organization of ventilation system of railway inside air conditioned passenger cars. [D].Shan'xi:Xi'an Transportation University,2001.

[7]Liu Zhanfeng,Songli etc”Auto air conditioner” [M]Beijing:Beijing University Pressure,2001. 